\title{
Fuzzy Cascade Control Research of Superheated Temperature
}

\author{
Ying-hui Xie ${ }^{1,}{ }^{*}$, and Xiao-qiu Liu ${ }^{2}$ \\ ${ }^{1}$ School of Electrical \& Information Engineering, Hunan International Economics University, \\ Changsha, Postcard:410205, China \\ ${ }^{2}$ School of Electrical \& Information Engineering, Hunan International Economics University, \\ Changsha, Postcard:410205, China \\ ${ }^{*}$ Corresponding author
}

Keywords: Delay System, Fuzzy Control, Superheated Temperature, Control System

\begin{abstract}
To the characteristics of the large delay and uncertainty of superheated temperature, a new cascade control system is presented based on control's history. Based on the analysis of the control objects'dynamic characteristics, historical control information(substituting for the deviation change rate)is used as the basis for decision-making of the fuzzy control. Therefore, the changing trend of the controlled variable can be accurately reflected. Furthermore, a proportional component is introduced, the advantages of PID and fuzzy controllers are integrated, and the structure weaknesses of conventional fuzzy controllers are overcome. Simulation shows that this control method can effectively reduce the adverse impact of the delay on control effects and, therefore, exhibit strong adaptability by comparing the superheated temperature control system by this controller with PID and conventional fuzzy controllers.
\end{abstract}

\section{Introduction}

The steam temperature regulation in superheating systems is widely discussed as an interesting problem in thermal power plants control. The economical advantages of steam temperature regulation can be expressed in the terms of performance and safety enhancement of the power plants, which are needed to meet the requirements of control quality. In order to attain the maximum efficiency of turbines and protect them against premature elements, the temperature deviations from design values have to be kept small [1].

Distributed structure, nonlinear behavior and time-varying dynamics of once-through boiler reverse to load variation may cause that attaining of a tight control would be a difficult challenge. Furthermore, declining the reliability and performance of aged thermal power plants could potentially increase the complexity of control systems, where conventional control is no longer sufficient for superheat temperature control [2].

In the recent years, fuzzy logic control systems (FLC) are widely used to handle complicated streams in presence of uncertainties and nonlinearities. Various applications of the FLC for temperature regulation in industrial processes are reported in literature. Most fuzzy controllers are develop only based on the error (e) and rate of error changes (e) as inputs for handling nonlinear systems [3]. However, these controllers are not able to distinguish the operating region and make an appropriate control action with respect to operating conditions [4]. In order to overcome the problem, different types of adaptive fuzzy logic controller such as self-tuning and self-organized controllers have been developed [5,6]. Using a nonlinear predictive controller designed on Takagi-Sugeno fuzzy model Is another method proposed for controlling the steam temperature in the literature $[7,8]$.

Currently, most control systems of superheated temperature adopt cascade control as shown in Fig.1.This kind of system is simple in structure and easy to execute. However, superheated temperature is a typical time-variable, non-linear delay system. Thus it is hard for a cascade control system to attain a satisfactory control performance. 


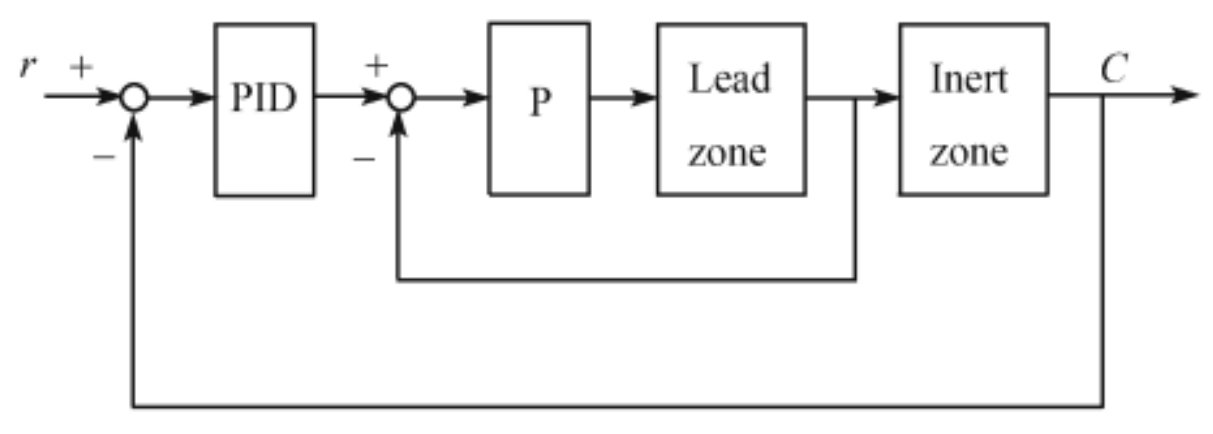

Figure 1. Diagram of cascade control system

Fuzzy control,which needs no exact mathematical model,has achieved partial success in controlling superheated temperature. However,the weaknesses of a traditional fuzzy controller are obvious.First,fuzzy rules are composed of three linguistic variables, which are,deviation,deviation change rate, and control variable.Both fuzzy control based on that structure and PID control are "control after the event",so it is not ideal to control the large delay object.Second,the control object with delay(the traditional fuzzy PI controller) has the defect of "gain-role change", which affects control quality.

To solve the above problems in the control system of superheated temperature, a new fuzzy control method that can be applied to large delay systems is proposed. According to this method, the fuzzy cascade control based on control's history for superheated temperature is formed.

\section{BCH Fuzzy Controller}

Delay system analysis : The pure delay time is denoted by $\tau, \mathrm{u}^{*}(\mathrm{t})=\mathrm{u}(\mathrm{t}-\tau)$, and $\mathrm{w}(\mathrm{t})$ represents the unit impulse response of non-delay object.The delay object is usually described in Fig.2

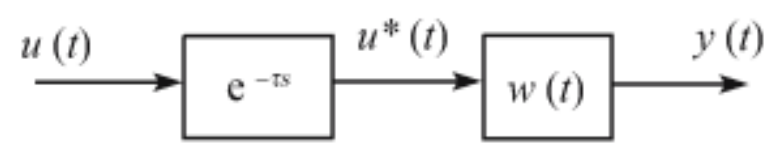

Figure 2. Diagram of delay system

According to the convolution theorem, the analogous features can be derived

Structure of BCH fuzzy controller : It is known from Fig.2 that at time t,the control signal $u(t)$ designed for $\mathrm{w}(\mathrm{t})$ and $\mathrm{y}(\mathrm{t})$ will function at $\mathrm{w}(\mathrm{t})$ after $\tau$.However, the output $\mathrm{y}(\mathrm{t}+\tau)$ after $\tau$ time is different from the output $\mathrm{y}(\mathrm{t})$ designed for $\mathrm{u}(\mathrm{t})$, thus $\mathrm{u}(\mathrm{t})$ cannot control the system at this particular time. Therefore, the trend of the output after $\tau$ must be considered when designing control signal $u(t)$.

In general, the deviation and the deviation change rate are used as two input variables in a two-dimensional fuzzy controller. The deviation change rate only indicates the variety of output at time $t$,but cannot do that effectively at time $(t+\tau)$. If this signal is still taken as input variable of the fuzzy controller, the deduced control signal is usually inapplicable to system state after $\tau$.

$\Delta y(t+\tau)$ is closely related to the output history of the controller. Thus, $\Delta u \tau$ can be chosen as one of the input variables. But $\Delta u \tau$ cannot totally represent the output characteristic after $\tau$. Here, the exact model without control object is adopted, i.e. $\Delta u \tau$ is used as input of fuzzy controller,instead of the deviation change rate, which is shown in Fig.3. The control variable fitted for system state after $\tau$ can be obtained. It is helpful to improve the control effect for the delay object. 


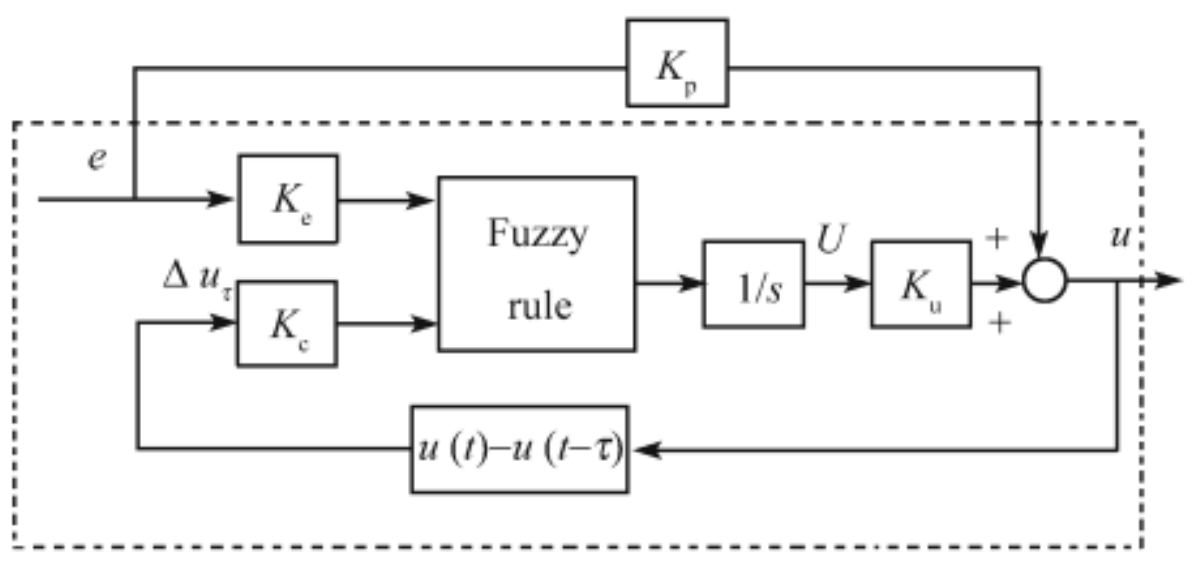

Figure 3. Diagram of fuzzy control system based on control's history

\section{PBCH Fuzzy Controller}

Structure Defect of FZ-PI : Fuzzy controller output as increment is defined as fuzzy controller PI(FZ-PI) as shown in Fig.4. The FZ-PI controller bares some similarities to the PI controller. The output increment of the PI controller can be expressed as

$$
\Delta \mathrm{u}_{\mathrm{PI}}=\mathrm{K}_{\mathrm{I}} \mathrm{E}(\mathrm{t})+\mathrm{K}_{\mathrm{P}} \mathrm{E}(\mathrm{t})
$$

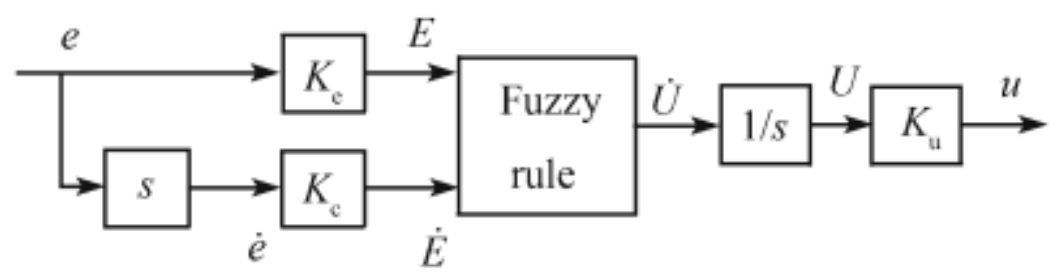

Figure 4. Diagram of FZ-PI controller

The output increment of FZ-PI controller can be expressed as

$$
\Delta \mathrm{uFZ}-\mathrm{PI}=\mathrm{F}(\mathrm{E}(\mathrm{t}), \grave{\mathrm{E}}(\mathrm{t}))=\mathrm{K}^{\prime} \mathrm{IE}(\mathrm{t})+\mathrm{K}^{`} \mathrm{P} \grave{\mathrm{E}}(\mathrm{t})
$$

Where $K_{P}, K_{I}$ are called proportional gain and integral gain respectively, which is shown as nonlinear function. In fact,the fuzzy PI controller from Eq.(2) probably has a function defect, because the deviation change rate, from 0 to $\tau$, is always $0(\mathrm{e}=0)$. This shows that the output of the controller is actually provided by integral action. In other words, during the time, this action mainly represents proportion action, i.e. "gain-role change" on the delay. According to the physical property of the PID controller, when the error is large, proportionate action should be large to speed up the response. When the error is small, integral action should be large to reduce the steady state error. Based on the analysis, the characteristics of this fuzzy PI controller at least cannot fit for physical property of the PID controller. So the effects of the gain-role change" makes it difficult to adjust the system's integral gain, and brings a bad effect to control performance.

Structure of PBCH fuzzy controller : The position types of the PI controller and FZ-PI controller can be described as:

$$
u P I=K p e(t)+K I \int t 0 e(t) d t
$$




$$
\text { uFZ-PI= Kuftof(e(t),è(t))dt }
$$

For the PI controller, when the control object shows deviation, according to the first item on the right of Eq.(3), the control variable will be obtained. Based on this, the control variable can adjust gradually from Eq.1), and the required control variable will be finally achieved. However, for the FZ-PI controller, the control variable only increases gradually from 0 . Looking from this aspect, this controller has more characteristics of I controller, which is the cause of "the gainrole change". In general, for the traditional PID controller,increasing the KP will speed up the response. For control objects with delay, in the initial stage, the KP is small, so overshoot is small in the control process. However, the slow variety rate of control variable will lead to a longer regulating time.

The mentioned fuzzy control based on control's history can, to some degree, lessen the defects of "gain-role change", but not radically. After comparing Eq. (3) with Eq.(4), KP is introduced into the $\mathrm{BCH}$ controller as shown in Fig.3. The integration of the speediness of the PID controller and the stability and adaptability of fuzzy control will speed up the response.

\section{Simulation}

Because of the larger heat inertia in superheated temperature, in step response curve, the hysteresis characteristic is obviously demonstrated.

In order to demonstrate the validity of $\mathrm{PBCH}$ fuzzy controller, the simulation of the superheated temperature control system of $600 \mathrm{MW}$ (Model 1) and $300 \mathrm{MW}$ (Model 2) is performed.

Model 1 (100\%load) and Model 2 in Table 1 are simulated respectively. In normal cascade PID control, the control parameter of the outer/inner controller adopts the optimal control parameters expressed in Table 2. With the inner controller unchanged, the outer controller adopts a traditional FZ-PI fuzzy control and $\mathrm{BCH}, \mathrm{PBCH}$ fuzzy control mentioned in this paper to do simulation for superheated temperature.

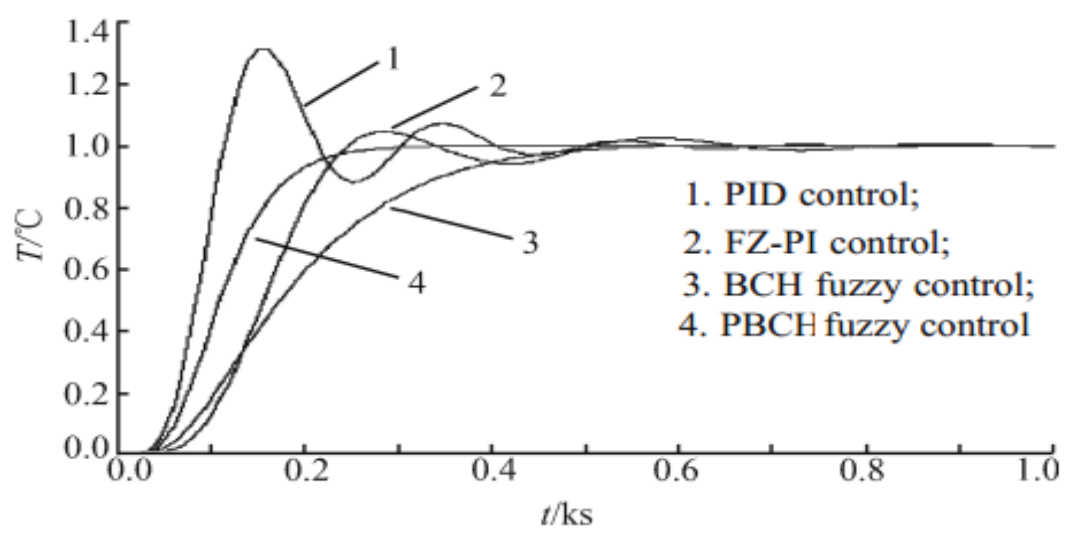

Figure 5. Responses of model 1 under 100\% load

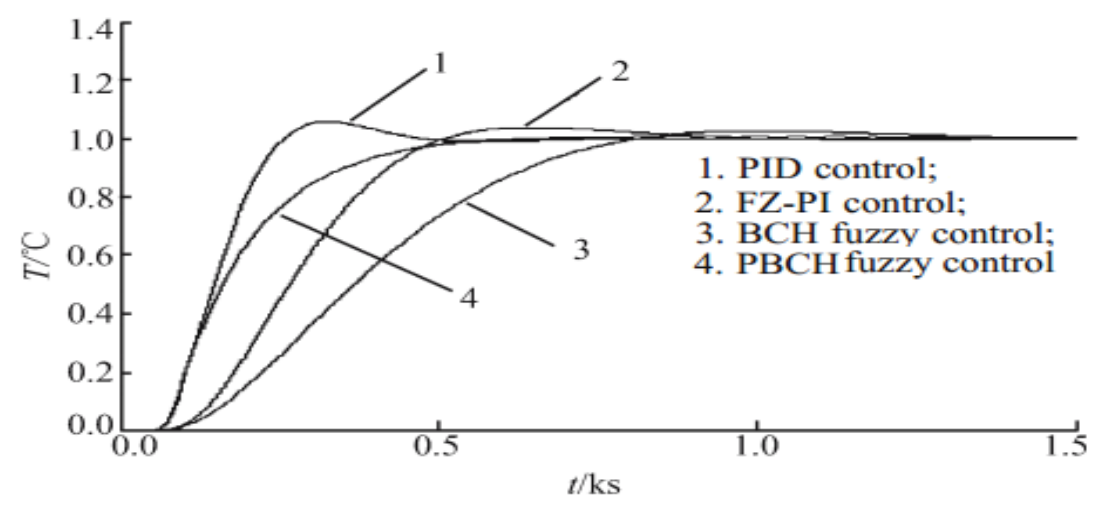

Figure 6. Responses of model 2 
When designing a PBCH fuzzy controller, both the inner controller and the object in the inert zone may be defined as generalized control object. A generalized control object can adopt the model structure of first order plus pure delay usually used in the project. The parameters of the model and the numerical value of $\tau$ can be ascertained by the recursive least squares (RLS) identification based on step response. Such $\tau$ is not very exact, but because the parameter of the PBCH fuzzy controller is well adapted to pure delay time well, it does not have much impact on the control efficiency. $\Delta u \tau$ in the PBCH fuzzy controller bares the same function to deviation change rate in the traditional fuzzy controller,so the fuzzy rule base in the fuzzy controller can succeed to Table 2 Tuning values of PID control Model Inner controller Outer controller the one in the traditional fuzzy controller, without much modification.

It can be seen from Figs. 5 and 6 that the cascade PID control usually causes larger overshoot. The traditional FZ-PI fuzzy control can reduce some overshoot, but will lead to longer regulating time due to the defects. The PBCH fuzzy control strategy reduces the negative effect of a large delay, and adding the proportional gain can speed up the response and reduce the overshoot. As a result, the whole control process, with its good control effect, is obviously much better than the two controllers mentioned previously.

In fact, it is hard to obtain exactly the feature parameters in the boiler steam temperature model (such as $\mathrm{t}$ ). Under different operating conditions, it is possible that the object characteristic vary. To test the adaptability of the $\mathrm{PBCH}$ cascade fuzzy control system, with the controller's parameters unchanged, Model 1 with 75\%load and 50\%load is used instead of the previous model with $100 \%$ load. Thus, the delay time of the inert zone in Model 2 is changed from 53 to $150 \mathrm{~s}$, thus the step dynamic response of the control system can be obtained.

The result of the simulation shows that, for the normal PID control system and the FZ-PI fuzzy control system, with the extension of delay time, the overshoot will increase, the response will slow down and control effect will finally worsen and become unstable. Whereas, in a PBCH fuzzy control system, $\Delta u \tau$ substitutes for the deviation change rate as input of the fuzzy controller, so when a larger variety of the object causes lager differences, $\Delta u \tau$ still demonstrates the changing trend of setting output well. Therefore, the overshoot does not vary much with increasing delay time, and has better adaptability compared with the normal PID control system and the FZ-PI fuzzy control system.

\section{Conclusions}

1. The PBCH fuzzy controller in this paper keeps the main merits of a normal fuzzy controller. But, by bringing in the control history data into the decision process of the fuzzy controller, it exhibits ideal control and adaptability, with much consideration of correlation between output after delayed and control signal, which provides a new control method for the autocontrol of large delay processes.

2. Based on previous fuzzy control models, the introduction of proportion factor integrates the advantages of a PID controller and the fuzzy controller, thus making this fuzzy control system correspond accordingly between the speed of the controller and the stability of the control system.

3. For superheated temperature, compared with the present cascade control system, the PBCH fuzzy cascade control system is proposed in this paper needs little improvement to the control system, and thus can be effectively carried out.

\section{References}

[1] R.N. Silva, P.O. Shirley, J.M. Lemos, and A.C. Goncalves, “Adaptive regulation of super-heated steam temperature: a case study in an industrial boiler," Control Engineering Practice, vol. 8, pp. 1405-1415, 2000.

[2] A. Ghaffari, A.R. Mehrabian and M. Mohammad-Zaheri, "Identification and control of power plant de-superheater using soft computing techniques," Engineering Applications of Artificial Intelligence, vol. 20, pp. 273-287, 2007. 
[3] A.S. Lopez, G.A. Figueroa, and A.V. Ramirez, "Advanced control algorithms for steam temperature regulation of thermal power plants," Electrical Power and Energy Systems, vol. 26, pp. 779-785, 2004.

[4] S.J. Qin, and G. Borders, "A multi-region fuzzy logic controller for nonlinear process control," IEEE Transactions on Fuzzy Systems, vol. 2, pp. 74-81,1994.

[5] I. Rojas, H. Pomares, J. Gonzalez, L.J. Herrera, A. Guillen, F. Rojas, and O. Valenzuela, "Adaptive fuzzy controller: application to the control of the temperature of a dynamic room in real time," Fuzzy Sets and Systems, vol. 157, pp. 2241-2258, 2006.

[6] C.J. Lin, “A GA-based neural fuzzy system for temperature control,” Fuzzy Sets and Systems, vol. 143, pp. 311-333, 2004.

[7] G. Karer, G. Mušip, I. Škrjanc, and B. Zupanpip, "Hybrid fuzzy model-based predictive control of temperature in a batch reactor," Computers and Chemical Engineering, vol. 31, pp. 1552-1564, 2007.

[8] M. He, W.J. Cai, and S.Y. Li, "Multiple fuzzy model-based temperature predictive control for HVAC systems," Information Sciences, vol. 169, pp. 155-174, 2005. 